\title{
A Case of Fabry Cardiomyopathy
}

\author{
Jong Chun Nah, MD11, Woo-Shik Kim, MD², Wook-Hyun Cho, $\mathrm{MD}^{1}$, Suk-Koo Choi, MD¹, \\ Han-Wook Yoo, MD³, Toshihiro Takenaka, $\mathrm{MD}^{4}$ and Chuwa Tei, MD ${ }^{4,5}$ \\ ${ }^{1}$ Division of Cardiology, Department of Internal Medicine, Seoul Paik Hospital, Inje University Medical College, Seoul, \\ ${ }^{2}$ Department of Internal Medicine, School of Medicine, Kyung Hee University, Seoul, \\ ${ }^{3}$ Department of Pediatrics, University of Ulsan College of Medicine, Asan Medical Center, Seoul, Korea \\ ${ }^{4}$ Division of Cardiac Repair and Regeneration, Graduate School of Medical and Dental Sciences, Kagoshima University, Kagoshima, \\ ${ }^{5}$ Department of Cardiovascular, Respiratory and Metabolic Medicine, Graduate School of Medical and Dental Sciences, \\ Kagoshima University, Kagoshima, Japan
}

\section{ABSTRACT}

In the absence of hypertension, hypertrophic cardiomyopathy is the most common cause of left ventricular hypertrophy (LVH). However, it has been reported that up to 3\% of males with unexplained LVH have Fabry disease, an X-linked disorder of glycophospholipid metabolism that is due to a deficiency in the lysosomal enzyme $\alpha$ galactosidase A ( $\alpha$-Gal A). A 44-year-old man was admitted to our hospital with palpitations. He had a history of chronic renal failure diagnosed at age 33 followed by kidney transplantation performed at our institution 2 years later, as well as long-standing hypohidrosis. His medications included prednisolone (5 mg daily), mycophenolate mofetil (1,000 mg, bid), and cyclosporine (150 mg, bid). On hospital day two, an echocardiogram demonstrated increased left ventricular wall thickness (septal wall thickness of $28 \mathrm{~mm}$, posterior wall thickness of $20 \mathrm{~mm}$ ). Diastolic dysfunction was noted on transmitral flow patterns and tissue Doppler imaging. The patient was found to have low plasma $\alpha$-Gal A activity. A previously reported H46R missense mutation was detected in his $\alpha$-Gal A gene and the patient was subsequently diagnosed with Fabry disease. (Korean Circ $\mathbf{J}$ 2009;39:335-339)

KEY WORDS: Fabry disease; Alpha-Galactosidase A; Cardiomyopathies.

\section{Introduction}

Fabry disease is a rare $\mathrm{X}$-linked disorder caused by mutations in the GLA gene encoding the lysosomal enzyme $\alpha$-galactosidase A ( $\alpha$-Gal A). It results in a failure to catabolize lipids containing $\alpha$-D-galactosyl moieties and subsequent progressive intracellular accumulation of glycosphingolipids in various tissues, including skin, kidneys, vascular endothelium, ganglion cells of the peripheral nervous system, and heart. The disorder is caused by mutations in the $\alpha$-Gal A gene located in the $\mathrm{X}$ ch-

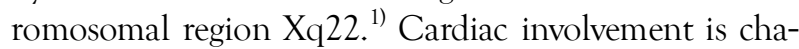
racterized by progressive left ventricular hypertrophy (LVH) that mimics the morphologic and clinical features of hypertrophic cardiomyopathy (HCM), which is an inherited autosomal dominant disease that is caused by mutations

Received: March 23, 2009

Accepted: June 1, 2009

Correspondence: Woo-Shik Kim, MD, Department of Internal Medicine, School of Medicine, Kyung Hee University, 1 Hoegi-dong, Dondaemungu, Seoul 130-702, Korea

Tel: 82-2-958-8169, Fax: 82-2-958-8160

E-mail:kkabee@dreamwiz.com in sarcomeric genes. ${ }^{23)}$ Since it has been reported that enzyme replacement therapy (ERT) is effective in reducing and clearing glycosphingolipid accumulation with subsequent improvement in cardiac function, early recognition of Fabry disease has become increasingly relevant. $^{45)}$

Here we report a case of Fabry disease which was first suspected based upon echocardiographic findings of $\mathrm{LVH}$.

\section{Case}

A 44-year-old man with no history of hypertension or diabetes mellitus was admitted with sudden onset palpitations of 2 hours duration. He had been diagnosed with end-stage renal disease (ESRD) of unknown etiology at age 33 followed by renal transplantation at age 35 . Since that time, he had been treated with oral immunosuppressive agents, including prednisolone (5 mg daily), mycophenolate mofetil $(1,000 \mathrm{mg}, \mathrm{bid})$ and cyclosporine (150 mg, bid).

Vital signs upon arrival included a blood pressure of 150/90 $\mathrm{mmHg}$ and a regular rapid pulse rate of $150 \mathrm{bpm}$. 
The initial electrocardiogram (ECG) revealed a narrow QRS tachycardia of approximately $150 \mathrm{bpm}$ with absent $\mathrm{P}$ waves (Fig. 1A). The patient was diagnosed with reentrant paroxysmal supraventricular tachycardia (PSVT) and was treated with intravenous adenosine. Following normalization of rhythm, the ECG showed LVH with a strain pattern, ST-T changes in leads II, V3, and V4,
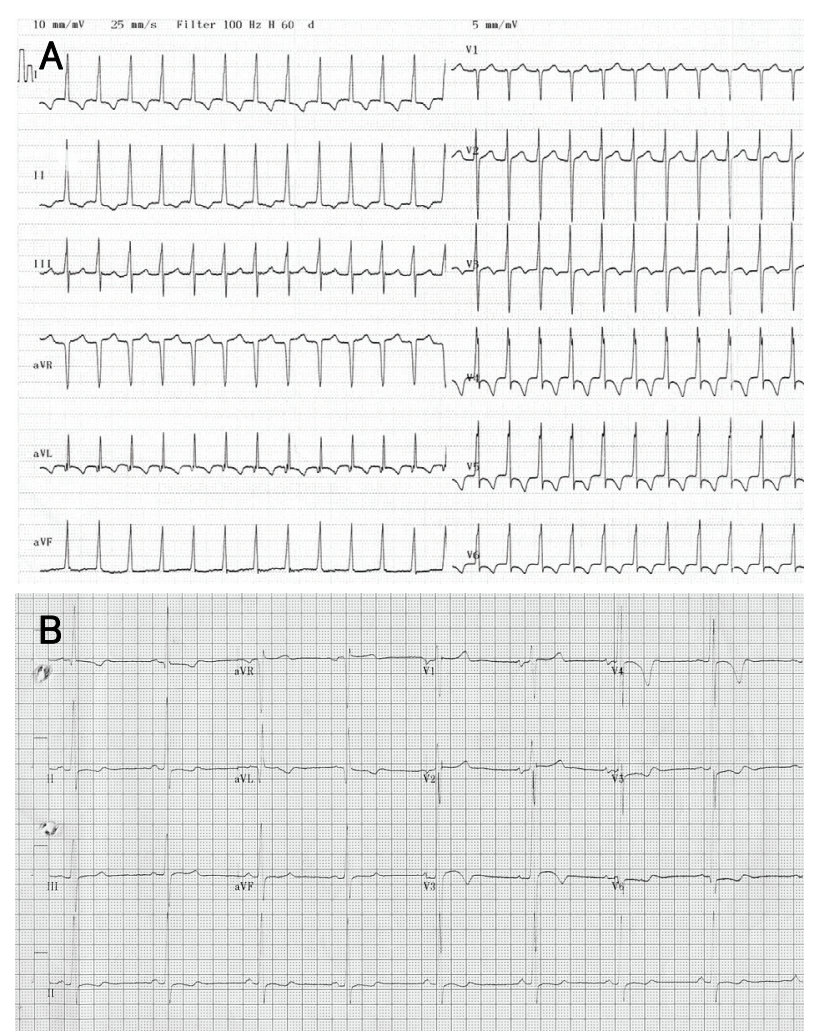

Fig. 1. Electrocardiography (ECG). The initial ECG (A) showed a narrow $Q R S$ tachycardia with absent $P$ waves. After chemical conversion, the ECG (B) showed left ventricular hypertrophy with a strain pattern, ST-T changes in leads II, V3, and V4 and a short PR interval. and a short PR interval (Fig. 1B). Chest radiography demonstrated mild cardiomegaly (Fig. 2). Laboratory studies revealed that hemoglobin was $13.3 \mathrm{~g} / \mathrm{dL}$, creatinine $1.7 \mathrm{mg} / \mathrm{dL}$, BUN $27 \mathrm{mg} / \mathrm{dL}$, sodium $134 \mathrm{mEq} / \mathrm{L}$, and potassium $4.1 \mathrm{mEq} / \mathrm{L}$. On hospital day two, transthoracic echocardiography revealed severe concentric $\mathrm{LVH}$ (septum $28 \mathrm{~mm}$, posterior wall $20 \mathrm{~mm}$ ) without left ventricular outflow tract obstruction, mimicking nonobstructive HCM (Fig. 3). Systolic left ventricular function was preserved but diastolic dysfunction was present. Pulsed-wave Doppler recording of mitral inflow revealed a phase resembling a normal diastolic filling pattern, with an E/A ratio of 1.6 (Fig. 4A). The mitral annulus velocity was obtained from the septal side of the mitral an-

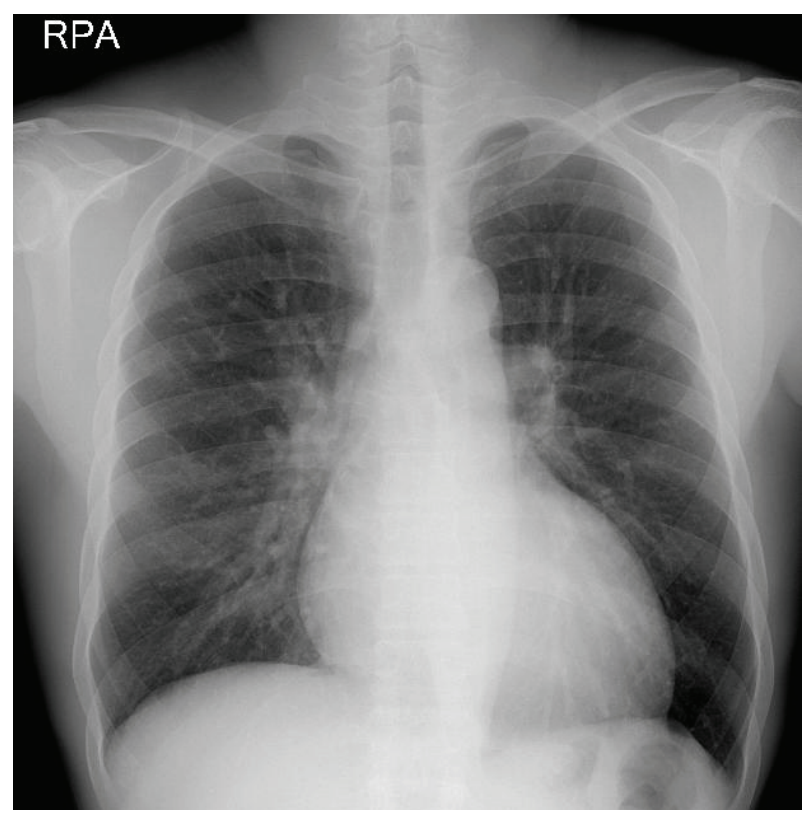

Fig. 2. Chest radiography. Chest radiography revealed mild cardiomegaly.
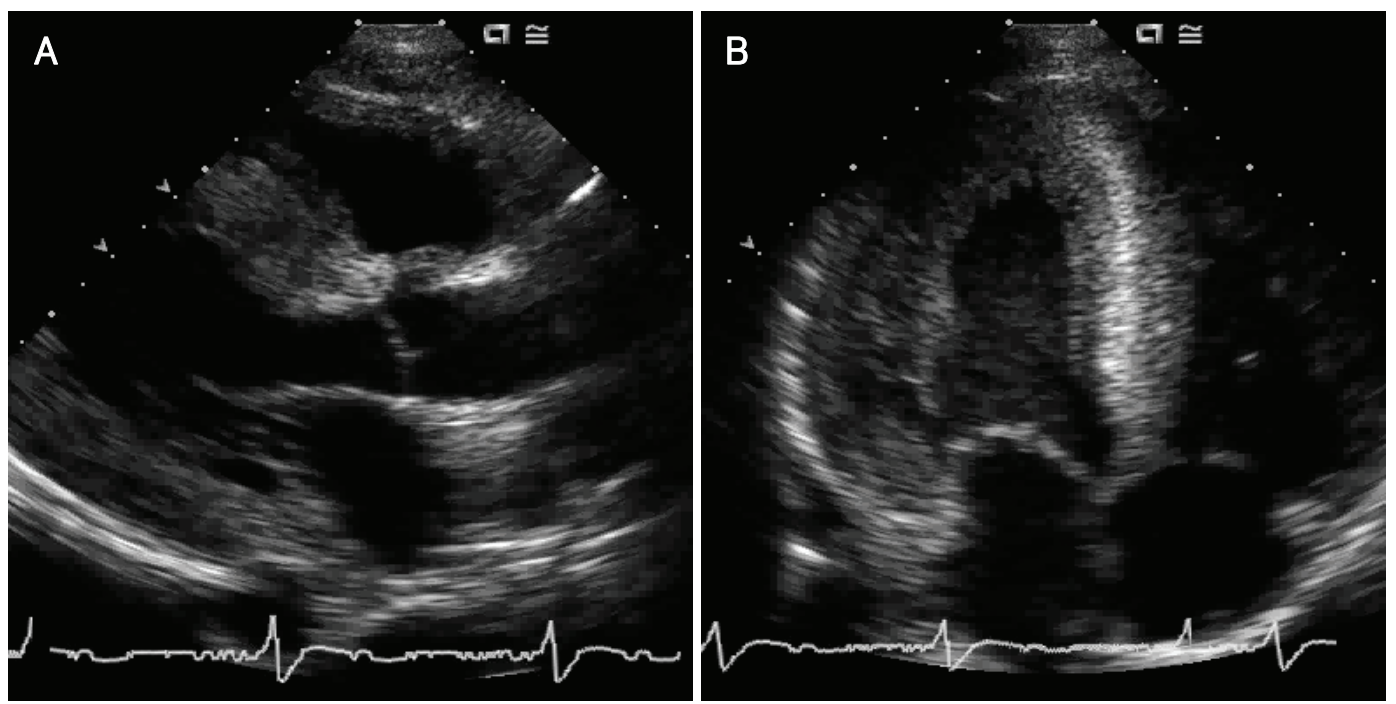

Fig. 3. 2-D echocardiography. Severe concentric left ventricular hypertrophy is shown in a parasternal long-axis view (A) and four-chamber view (B). 

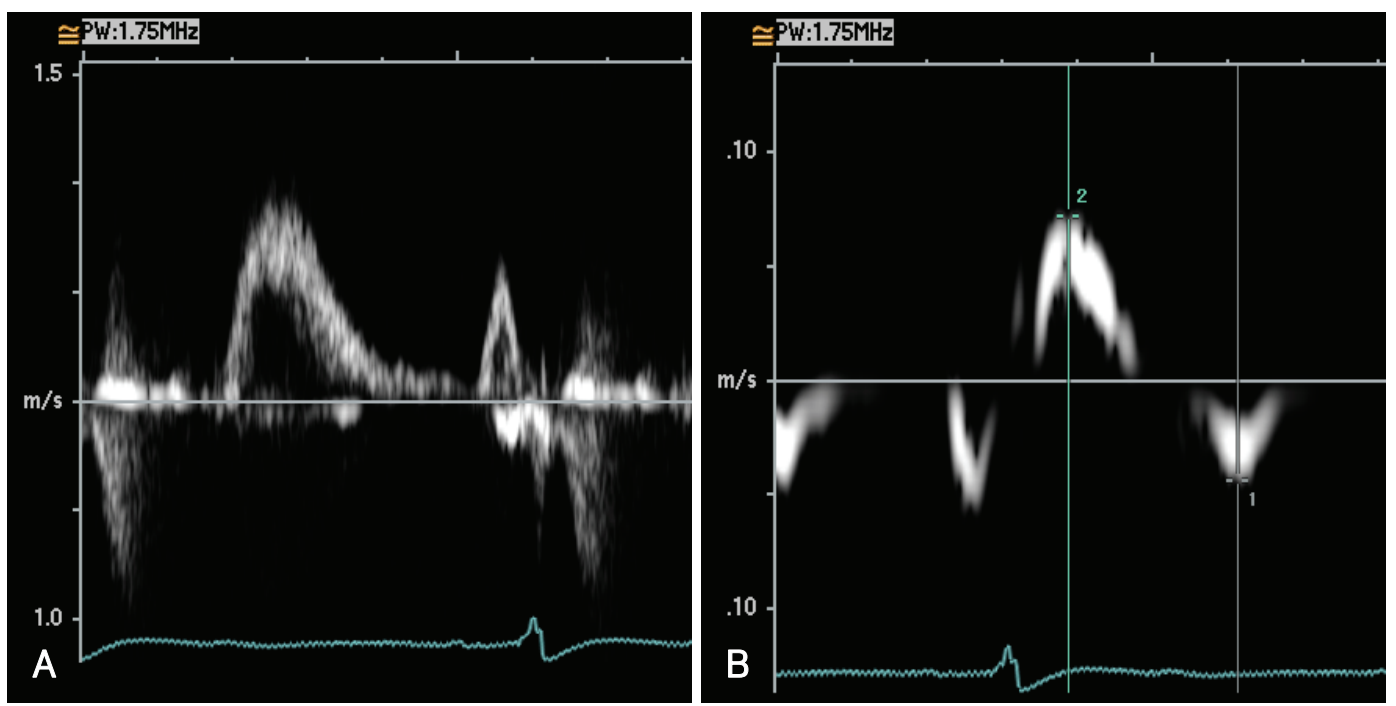

Fig. 4. Pulse-waved Doppler echocardiography (A) and tissue Doppler echocardiography (B). Decreased mitral annulus velocities and increased E/Ea ratio are seen, suggesting a pseudonormal pattern.

PCR-Sequence analysis

Normal

G G G C T G GC GCACT G G G A C GC T

Patient
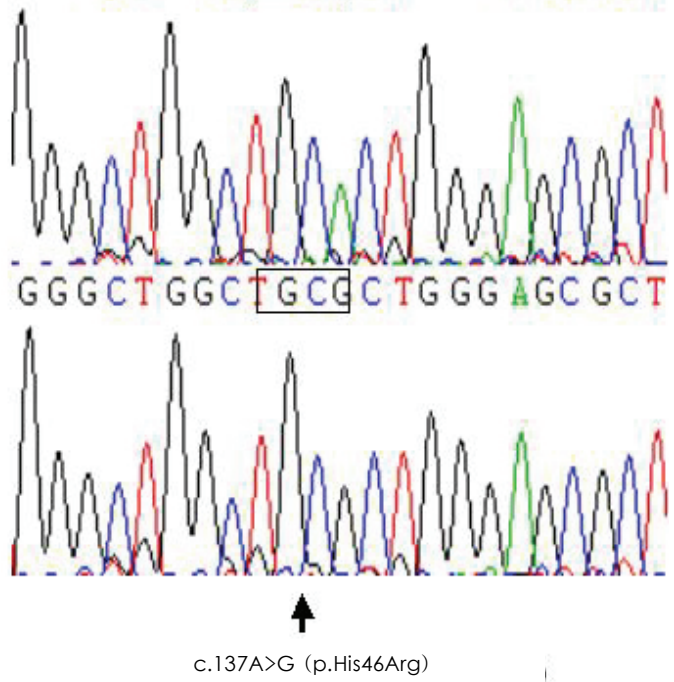

Fig. 5. DNA analysis. Sequent analysis of polymerase chain reaction (PCR)-amplified genomic DNA showing a hemizygous single nucleotide transition $(C 137 A>G)$ leading to a missense mutation with arginine substituting for a histidine at residue 46.

nulus using Doppler tissue imaging to distinguish a true normal from a pseudonormal pattern (Fig. 4B). The early diastolic tissue Doppler velocity $(\mathrm{Ea})$ and the $\mathrm{E} / \mathrm{Ea}$ index were $0.5 \mathrm{~cm} / \mathrm{s}$ and 22 , respectively, indicating increased LV filling pressure and a pseudonormal pattern. Since infiltrative diseases could not be ruled out based on clinical and echocardiographic findings, a careful history was obtained, revealing chronic hypohidrosis and heat intolerance. Fabry cardiomyopathy was thus suspected due to its typical history of early onset ESRD and renal transplantation prior to 40 years of age as well as hypohidrosis and heat intolerance. This diagnosis was confirmed by demonstration of a low plasma $\alpha$-Gal A activity of 0.17 nmoles $/ \mathrm{hr} / \mathrm{mL}$ (normal mean, $8.5 \pm 2.4$ $\mathrm{nmol} / \mathrm{hr} / \mathrm{mL}$ ). A His46Arg missense mutation in the $\alpha$-Gal A gene, which resulted in the substitution of arginine for histidine, was subsequently identified from lymphocyte DNA (Fig. 5). A transplant renal biopsy was performed because chronic renal allograft rejection was suspected given the increased serum creatinine level and high resistive index of the transplanted kidney; however, the biopsy was unremarkable. Enzyme replacement therapy with recombinant $\alpha$-Gal A was started on an outpatient basis.

\section{Discussion}

Hundreds of inborn errors of metabolism have been reported and have been classified according to the type of metabolism involved. Lysosomal storage disorders, one of the major categories of inborn errors of metabolism caused by a deficiency of specific lysosomal enzymes, are generally classified according to the involved substrates as follows: sphingolipidoses, glycoproteinoses, mucolipidoses, mucopolysaccharidoses (MPSs), and many others. Cardiac disease is particularly prominent in lysosomal glycogen storage diseases (Pompe and Danon disease), MPSs and in glycosphingolipidoses (Fabry disease). Among lysosomal storage disorders, enzyme replacement therapy for the treatment of Gaucher disease, Pompe disease, Fabry disease and MPS I, II and VI has been approved in the United States. ${ }^{6}$

Fabry disease is an X-linked recessive inborn error of glycosphingolipid catabolism due to deficient or absent activity of the lysosomal enzyme, a-Gal A. Cardiac invo- 
lvement, consisting of progressive deposition of globotriaosylceramide $(\mathrm{Gb3})$ in myocardial cells leading to $\mathrm{LVH}$, is the most frequent cause of death. ${ }^{1)}$ Among patients with late-onset HCM, Fabry cardiomyopathy was diagnosed in up to $6 \%$ of men and up to $12 \%$ of women. ${ }^{788}$ Cardiac accumulation of $\mathrm{Gb} 3$ leads to thickening of the cardiac wall and may cause mitral valve prolapse, arrhythmia, heart attack, heart failure and stroke. Rarely, the heart may be the only organ involved in the so-called "cardiac variant," wherein patients present with a mild, late-onset disorder limited to the heart. ${ }^{910)}$

Here we report a case of Fabry cardiomyopathy with early onset ESRD and renal transplantation. To our knowledge, this is the first report of Fabry cardiomyopathy in Korea. The patient's clinical and echocardiographic findings were suggestive of an infiltrative disease as well as $\mathrm{HCM}$; however, $\mathrm{HCM}$ was thought to be more likely than infiltrative cardiomyopathy in which electrocardiography commonly exhibits a low voltage. ${ }^{11)}$ In addition, the patient had typical manifestations of Fabry disease, including hypohidrosis and heat intolerance, which were eventually diagnosed both biochemically and genetically. Mild left ventricular diastolic dysfunction is commonly present in the early stages of the disease; however, in advanced stages, systolic and severe diastolic dysfunction are present. ${ }^{12-14)}$ In our patient, transthoracic echocardiography revealed concentric $\mathrm{LVH}$ and stage 2 diastolic dysfunction. Upon initial presentation, the chief complaint was palpitations with an ECG indicative of PSVT. The prevalence of reported persistent atrial fibrillation, paroxysmal atrial fibrillation, and nonsustained ventricular tachycardia in Fabry disease are 3.9\%, 13.3\% and $8.3 \%$, repectively. ${ }^{15)}$ Following chemical conversion, the ECG revealed $\mathrm{LVH}$ with a strain pattern, ST-T changes in leads II, V3, and V4 and a short PR interval, which are all electrocardiographic findings common in most adult patients with Fabry disease. The short PR interval may have been the result of accelerated AV conduction rather than the presence of an accessory pathway. ${ }^{16)}$

Percutaneous biopsy of the transplanted kidney was performed to rule out chronic rejection. The findings on renal biopsy were normal, indicating that there was no evidence of recurrent Fabry disease in the graft. The risk of recurrence following renal transplantation has been reported to be less than $5 \% .{ }^{17}$ Both short- and longterm outcomes with kidney transplantation in ESRD patients with Fabry disease are reported to be good and comparable to those of kidney transplant recipients who developed ESRD from other causes. ${ }^{18)}$

Following discharge, our patient began enzyme replacement therapy with Fabrazyme (Agalsidase-beta). Two recombinant enzyme preparations of Agalsidase-alpha and Agalsidase-beta are now available for the treatment of Fabry disease. Several studies have reported the clearance of microvascular endothelial deposits of globotriao- sylceramide from the kidneys, heart and skin with subsequent improvement in neurological, renal and cardiac manifestations of the disease as well as in quality of life. ${ }^{34419120)}$ Therefore, early diagnosis is imperative for the modification of these manifestations and the disease course with enzyme replacement therapy.

\section{REFERENCES}

1) Desnick RJ, Ioannou YA, Eng CM. $\alpha$-Galactosidase A deficiency: Fabry disease. In: Scriver CR, Beaudet AL, Sly WS, eds. The Metabolic and Molecular Bases of Inherited Disease. New York: McGraw-Hill; 2001. p.3733-74.

2) Tanaka H, Adachi K, Yamashita Y, Toshima H, Koga Y. Four cases of Fabry's disease mimicking hypertrophic cardiomyopathy. $J$ Cardiol 1988; 18:705-18.

3) Jeong JW. Hypertrophic cardiomyopathy. Korean Circ J 2002; 32:7-14.

4) Eng CM, Guffon N, Wilcox WR, et al. Safety and efficacy of recombinant human a-galactosidase A: replacement therapy in $\mathrm{Fa}$ bry's disease. N Engl J Med 2001;345:9-16.

5) Weidemann F, Niemann M, Breunig F, et al. Long-term effects of enzyme replacement therapy on Fabry cardiomyopathy: evidence for a better outcome with early treatment. Circulation 2009;119: 524-9.

6) Burrow TA, Hopkin RJ, Leslie ND, Tinkle BT, Grabowski GA. Enzyme reconstitution/replacement therapy for lysosomal storage disease. Curr Opin Pediatr 2007;19:628-35.

7) Sachdev B, Takenaka T, Teraguchi H, et al. Prevalence of Anderson-Fabry disease in male patients with late onset hypertrophic cardiomyopathy. Circulation 2002;105:1407-11.

8) Chimenti C, Pieroni M, Morgante E, et al. Prevalence of Fabry disease in female patients with late-onset hypertrophic cardiomyopathy. Circulation 2004;110:1047-53.

9) von Scheidt W, Eng CM, Fitzmaurice TF, et al. An atypical variant of Fabry's disease with manifestations confined to the myocardium. N Engl J Med 1991;324:395-9.

10) Nakao S, Takenaka T, Maeda M, et al. An atypical variant of $F a$ bry's disease in men with left ventricular hypertrophy. $N$ Engl J Med 1995;333:288-93.

11) Cohen IS, Fluri-Lundeen J, Wharton TP. Two dimensional echocardiographic similarity of Fabry's disease to cardiac amyloidosis: a function of ultrastructural analogy? J Clin Ultrasound 1983; 11:437-41.

12) Linhart A, Palecek T, Bultas J, et al. New insights in cardiac structural changes in patients with Fabry's disease. Am Heart $J$ 2000;139:1101-8.

13) Kawano M, Takenaka T, Otsuji Y, et al. Significance of asymmetric basal posterior wall thinning in patients with cardiac Fabry's disease. Am J Cardiol 2007;99:261-3.

14) Takenaka $T$, Teraguchi $H$, Yoshida A, et al. Terminal stage cardiac findings in patients with cardiac Fabry disease: an electrocardiographic, echocardiographic, and autopsy study. J Cardiol 2008; 51:50-9.

15) Shah JS, Hughes DA, Sachdev B, et al. Prevalence and clinical significance of cardiac arrhythmia in Anderson-Fabry Disease. Am J Cardiol 2005;96:842-6.

16) Jastrzebski M, Bacior B, Dimitrow PP, Kawecka-Jaszcz K. Electrophysiological study in a patient with Fabry disease and a short PQ interval. Europace 2006;8:1045-7.

17) Hariharan S. Recurrent and de novo diseases after renal transplantation. Semin Dial 2000;13:195-9.

18) Obrador GT, Ojo A, Thadhani R. End-stage renal disease in pa- 
tients with Fabry disease. J Am Soc Nephrol 2002;13 (Suppl 2): S144-6.

19) Schiffmann R, Kopp JB, Austin HA 3rd, et al. Enzyme replacement therapy in Fabry disease: a randomized controlled trial. JAMA
2001;285:2743-9

20) Banikazemi M, Bultas J, Waldek S, et al. Agalsidase-beta therapy for advanced Fabry disease: a randomized trial. Ann Intern Med 2007; 146:77-86. 